\title{
Visualization of Flows in Curved Channels with a Moderate or High Rotation Speed
}

\author{
LIQIU WANG ${ }^{\mathrm{a}}$ and K. C. CHENG ${ }^{\mathrm{b}, *}$ \\ ${ }^{a}$ School of Mechanical \& Production Engineering, Nanyang Technological University, Nanyang Avenue, Singapore 639798; ${ }^{\mathrm{b}}$ Department \\ of Mechanical Engineering, University of Alberta, Edmonton, Canada T6G $2 G 8$
}

(Received June 1996; In final form August 1996)

\begin{abstract}
Flows in channels with streamwise curvature and spanwise rotation are visualized in terms of end-view near the exit of the test sections through injecting smoke into the flows. Two test sections are used, i.e. the rectangular channels with the aspect ratio of 1 and 10, respectively. The work focuses on visualization of Dean and Coriolis vortices under the effects of secondary instabilities and flows in the region with a relatively high rotation speed. The results show that the secondary instabilities cause the Dean and Coriolis vortices oscillating in various forms and the flows at high rotation speeds are controlled by the secondary instabilities rather than the primary instability. In particular, the secondary instabilities lead the flows to be unsteady and turbulent somewhat like the bursting flow in the turbulent boundary layers.
\end{abstract}

Keywords: Visualization, Rotating curved flow, Dean vortices, Coriolis vortices, Secondary instability

\section{INTRODUCTION}

Flows in rotating curved channels arise in a variety of practical processes. Examples are flows in centrifuges (Hochrainer [1971]), cooling channels of rotating machinery (Ito and Motai [1974], Miyazaki [1971, 1973] and Morris [1981]), particle separation devices (Lennartz et al. [1987], Papanu et al. [1986], Hoover et al. [1984], Stöber and Flachsbart [1969] and Kotrappa and Light [1972]) and rotating heat exchangers (Qiu et al. [1990]). Studies on the hydrodynamics in the rotating curved channels are, therefore, not only of considerable theoretical interest, but also of practical importance.

The physical model is illustrated in Fig. 1 (also see Fig. 3). Under the action of the pressure gradient along the channel axis, a viscous fluid is allowed to flow through a channel of rectangular cross section with width $\times$ hight of $(a \times b)$. The channel is uniformly curved around the axis $o^{\prime} z^{\prime}$ (streamwise curvature). At same time, the channel is rotating about that axis with a constant angular velocity $\Omega$ (spanwise rotation). The rotation can be positive or negative as shown in Fig. 1 in terms of angular velocity vector. If positive, the ro-

*Corresponding author. Tel.: 403-492-3598. Fax: 403-492-2200. 
tation direction is in the direction of the relative velocity of the fluid inside the channel. When the rotation is negative, however, the rotation direction is in the direction opposite to the relative velocity of the fluid. The radial, spanwise and streamwise directions are $(X, Y, \theta)$ respectively.

The streamwise curvature and spanwise rotation of the channel introduce centrifugal and Coriolis forces in the momentum equations which describe the relative motion of fluids with respect to the channel. Such body forces can stabilize the channel flow in some flow domain, and destabilize the flow in the other domains. In particular, the stabilizing or destabilizing effects due to curvature and rotation may either enhance or counteract each other depending on the direction of the rotation. This can be intuitively understood through a simple analysis of force directions. In the plane of the cross section, the centrifugal force always acts outwards in the positive $X$-direction. However, the Coriolis force may act in either positive or negative $X$-direction depending on the rotation direction. If the rotation is positive, it will act along the positive $X$-direction. When the rotation is negative, however, it will act along the negative $X$-direction. Therefore, we may expect a change in flow pattern as the rotation direction and rate change.

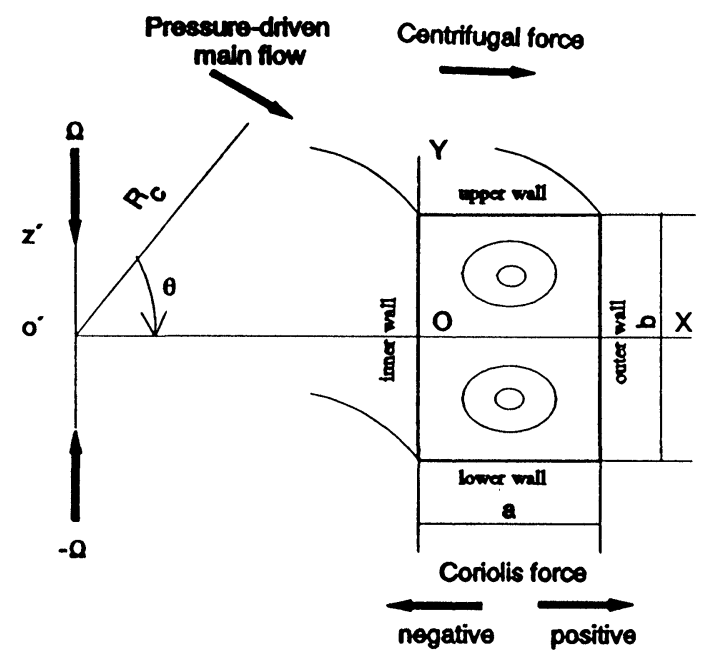

FIGURE 1 Flow in a channel with streamwise curvature and spanwise rotation.
For the case of positive rotation with a low rotation rate (Ro $\sim o(\sigma) ; R o$ is the rotation number, Eq. (2); $\sigma$ $=a / R_{c}$, the curvature ratio of the channel in Fig. 1), Miyazaki [1971, 1973] numerically analyzed the fully developed laminar flow in curved, rotating, circular and rectangular channels by a finite-difference method. The secondary flow revealed consists of one pair of counterrotating vortices in a plane perpendicular to the axis of the channel. The interaction of the secondary flow with the pressure-driven main flow shifts the location of the maximum streamwise velocity away from the center of the channel and in the direction of the secondary velocity in the central region of the channel. The effects of the rotation direction were not investigated by Miyazaki [1971, 1973]. Considering the channel to be a tube of circular cross sections with either positive or negative rotation, the fully developed laminar flow was investigated theoretically by Ito and Motai [1974], and Wang and Cheng [1996a]. The equations of motion were solved by a perturbation method for a small curvature and a low angular rotation rate of the tube. The inward Coriolis forces due to the negative rotation were found to cause the direction of the secondary flow to reverse by overcoming the outward centrifugal forces in the plane of the cross section. The flow reversal occurs by passing through a multi-pair vortex flow region where overall, the effect of the Coriolis force just neutralizes that of the centrifugal force. Such flow reversal and multipair vortex flow were also found in rectangular channels by a finite-volume numerical analysis (Wang and Cheng [1996b], and Wang 1995) and were experimentally confirmed by Wang and Cheng [1995].

For the case of moderate rotation in both positive and negative directions $(R o \sim o(1))$, the fully developed laminar flow was examined by Daskopoulos and Lenhoff [1990] for circular tubes and by Wang [1995], Wang and Cheng [1996b], and Selmi et al. [1994] for rectangular channels. The centrifugal and Coriolis forces were found to cause the centrifugal and Coriolis instabilities in the forms of streamwiseoriented roll cells (Dean and Coriolis vortices). The interaction between the two instabilities results in a complex flow structure. In particular, the appearance of the Dean and Coriolis vortices leads to a high in- 
flectional profile for streamwise velocity. Such a profile may be unstable with respect to streamwise wavy disturbances in accordance with the Rayleigh's inflection point criterion.

For a channel with an infinite span, Finlay et. al. [1988] and Finlay [1990] examined the secondary stabilities of the Dean vortices and the Coriolis vortices with respect to the streamwise wavy disturbances. They found that the instability leads to the development of undulating vortex flow and twisting vortex flow. As well, the stability of Dean vortices and Coriolis vortices subjected to two-dimensional spanwise periodic disturbances (i.e. Eckhaus stability) was examined numerically by Guo and Finlay [1991] for infinite span cross section. They found that the Eckhaus instability causes the vortex pairs to split apart or merge together. This motivates the first part of the present study to experimentally confirm these secondary instabilities and to expose the other possible secondary instabilities.

It appears that the literature on high rotation rates $(|R o|>3)$ is rather limited. For the case of the positive rotation with a high rotation rate, Ludwieg [1951] and Hocking [1967] employed the boundary layer approximation to theoretically examine the fully developed laminar flow in a curved channel with a square and a rectangular cross-section, respectively. They found that the secondary flow also consists of one pair of counter-rotating vortices in the cross plane. The streamwise velocity profile assumes a Taylor-Proudman configuration in the core region (Greenspan [1968]). While Ludwieg [1951] and Hocking [1967] appear to be the only works on the high rotation case, the validity of the boundary layer approximation has not been established for the flow with secondary flows. As a matter of fact, all the numerical simulations (Wang [1995], Wang and Cheng [1996b], Daskopoulos and Lenhoff [1990], Nandakumar et al. 1991, and Selmi et al. [1994]) failed to find stable flows if the rotation rate is high. This motivates the second part of the present work to experimentally visualize the flows in curved channels rotating at a higher rotation rate. Note that the rotating curved channels are usually operated at a high rotation rate in practical applications, and the results presented in this paper are believed to be not only of theoretical interest but also of practical importance.

In the present work, a smoke visualization apparatus is designed to visualize the flow in two curved channels rotating spanwise at a moderate or high rotation rate. The work mainly contains two parts. The first part is to experimentally visualize flow under the effect of the secondary instabilities of Dean and Coriolis vortices. The second part is to visualize flow at a high rotation rate. In addition, the primary instability analysis by Wang and Cheng [1995] is extended to the case with a high rotation rate. This leads to a conclusion that the flows at a high rotation rate are controlled by the secondary instability rather than the primary instability.

\section{EXPERIMENTAL APPARATUS AND TECHNIQUE}

A schematic diagram of the experimental apparatus is shown in Fig. 2. It consists of a test section, a rotating table with the rotating seal, an air supply system and a smoke generator.

The rotating table is driven by an electric motor with adjustable speed drive and the range of the rotating speed is $n=0 \sim 500 \mathrm{rpm}$. The rotational speed is measured by using an optical slot switch running on a disc with equally spaced 60 holes near its perimeter. The signal from the switch is fed to a Hewlett Packard HP 5314A Universal counter. With 60 holes in the disc, the frequency in $\mathrm{Hz}$ equals the rotational speed in rpm.

A second disc with a single hole provides the signal for firing the General Radio 1540 Strobolume by way of a delay generator/single flash flip-flop which allows visual observation using a slit light source with one flash per rotation and also permits a single, properly timed flash for photographing the whole secondary flow field.

The building compressed air is used as the fluid. The air flow rate is measured by a Meriam flow element with a calibrated differential pressure transducer. The smoke generated by burning Chinese in- 


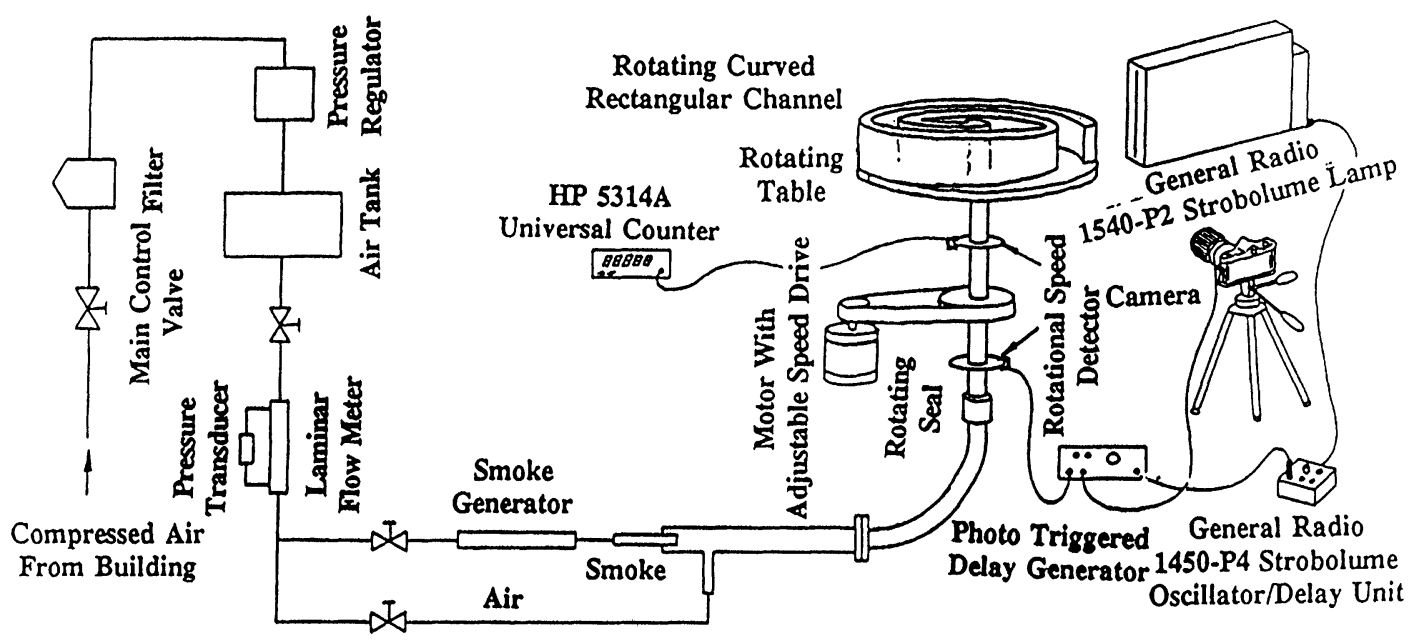

FIGURE 2 Schematic diagram of experimental apparatus.

cense sticks is injected through a dispersing tube before the test section as shown in Fig. 2. The very tiny smoke particle $\left(10^{-2} \sim 10^{-1} \mu \mathrm{m}\right)$, subjected to neglected gravitational and rotational buoyancy forces relative to the drag forces, marks fluid particle trajectories. The smoke patterns are photographed instantly near the exit of the test section $(\sim 0.8 \mathrm{~cm}$ from the exit) revealing the flow pattern. This provides an endview of the secondary flow pattern for an observer looking upstream into the channel cross-section. A Nikon FM2 single lens reflex camera and Kodak TMax black and white film P3200 are used.

Two test sections, denoted by $T s-A$ and $T s-B$, are shown in Fig. 3. The top view and the exit crosssection of $T_{s}-A$ are shown in Fig. 3(a). It consists of an entrance spiral square channel with axial length $0.85 \mathrm{~m}$ and a curved square channel $\left(270^{\circ}\right.$ bend with axial length of $1.2 \mathrm{~m}$ ) with a constant radius of curvature $R_{c}=25.4 \mathrm{~cm}$. The curved square channel has cross-section $5.08 \times 5.08 \mathrm{~cm}^{2}$, and is made from acrylic sheets. The air flows through a rotating straight tube (with an inside diameter of $4 \mathrm{~cm}$ ) along the axis of rotation, and then enters the spiral square channel before entering the test section. The flow near the exit of the channel is believed to be fully developed because of a ratio of the length over the width of 40.4 .
$T S$ - $B$, also made from acrylic sheets, consists of an entrance spiral rectangular channel with axial length $1.25 \mathrm{~m}$ and a curved rectangular channel $\left(270^{\circ}\right.$ bend $)$ with a constant radius of curvature $R_{c}=25.4 \mathrm{~cm}$. The top view and the exit cross section for $T s-B$ are shown in Fig. 3(b). The air flows through a rotating straight tube (with an inside diameter of $4 \mathrm{~cm}$ ) along the axis of rotation and then enters the spiral rectangular channel before flowing into the curved channel. With an entrance spiral channel length of $1.25 \mathrm{~m}$ and a subsequent curved rectangular channel (length $=1.2 \mathrm{~m}$ ) with a constant radius of curvature, the flow near the exit of the channel is also believed to be fully developed because the ratio of the length over the width of the channel is 96.5 .

\section{RESULTS AND DISCUSSION}

The end-view photographs are to be shown for $T s-A$ and $T s-B$ with spanwise direction vertical and radial direction horizontal. In each photograph, the convex (inner) wall is on the left and the concave (outer) wall is on the right. For a given test section, the flow is characterized by two parameters, namely, Reynolds 


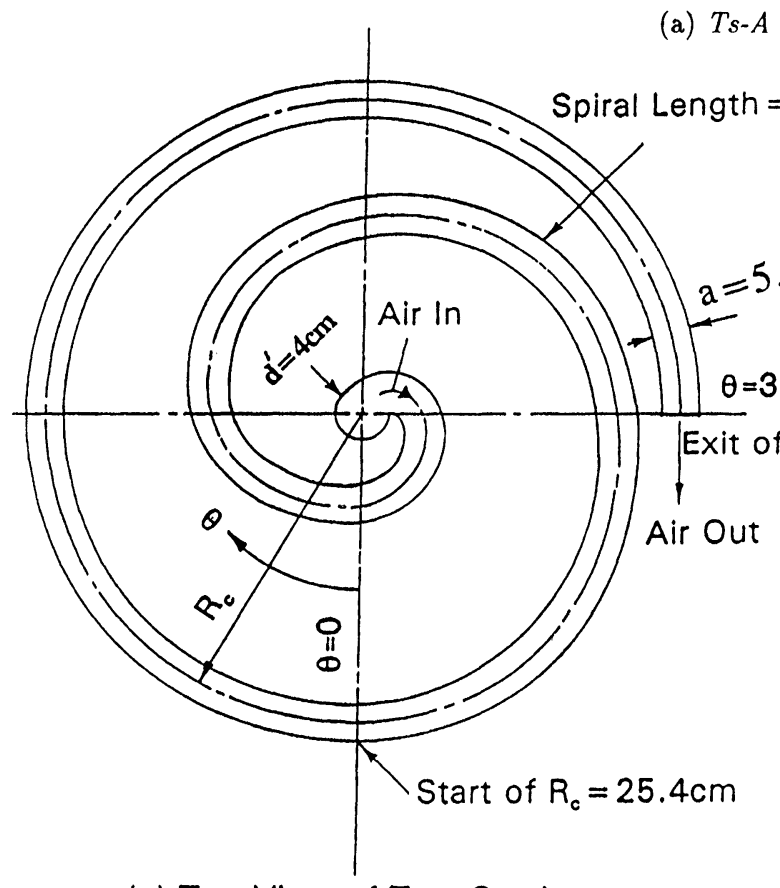

(a) Top View of Test Section

(a) $T s-A$

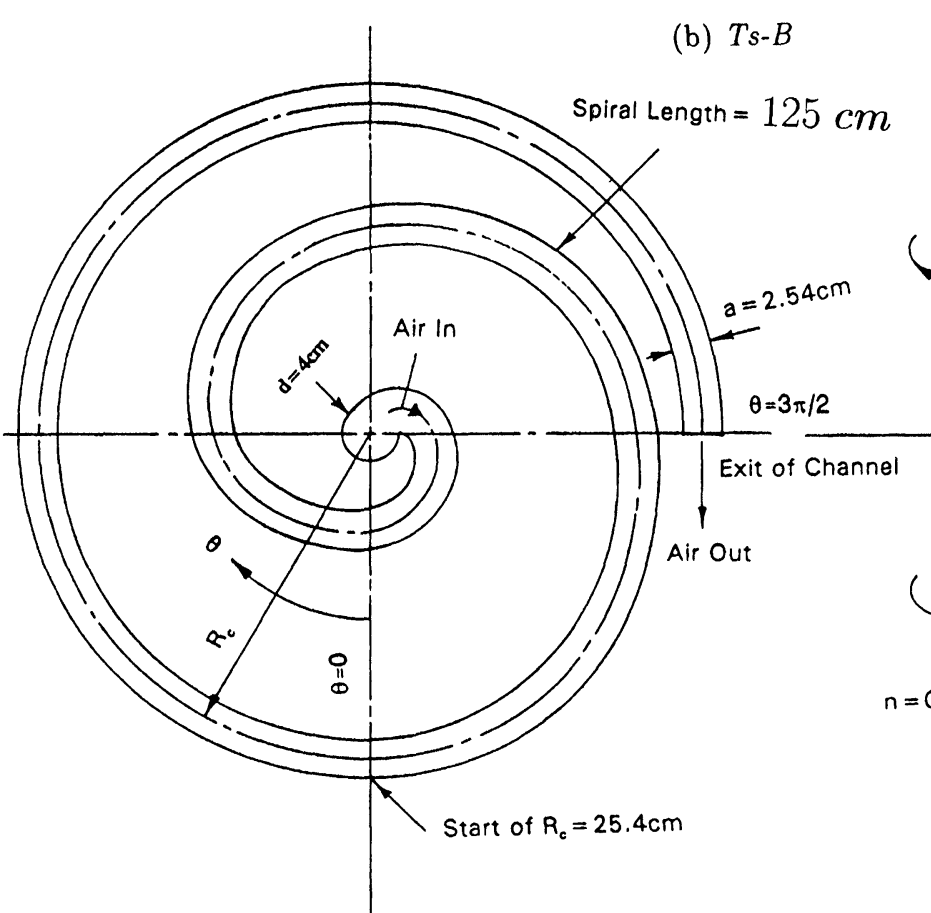

(a) Top View of Test Section (b) Channel Exit
$\Omega$

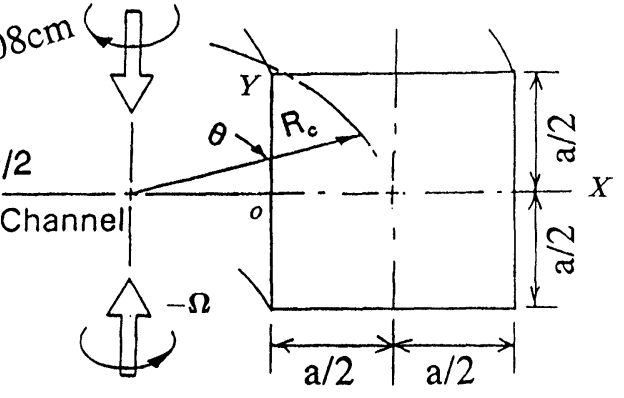

$\mathrm{n}=0-500 \mathrm{rpm}$

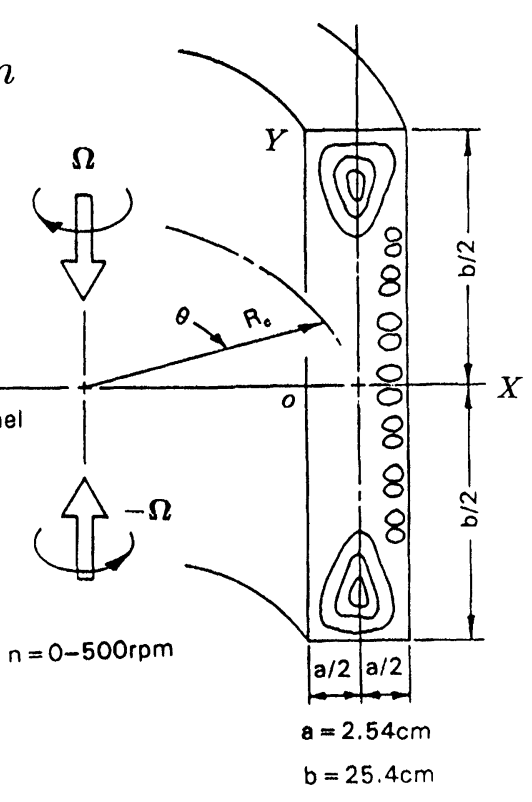

(b) Channel Exit

FIGURE 3 Two test sections. 
number $R e$ and rotation number $R o$ which are defined as

$$
\begin{gathered}
R e=\frac{W_{m} a}{v} \\
R o=\frac{\Omega a}{W_{m}}
\end{gathered}
$$

where $W_{m}, v, \Omega$ are the mean streamwise velocity, the kinematic viscosity of air and the angular rotation speed of the channel. The channel width $a$ is used as the characteristic length in $R e$ and $R o$. For a given set of $R e$ and $R o$, several photographs, each taken at different instant, are usually presented to show the time variation of the flow qualitatively. No attempt was made to specify the exact time intervals.

For $T s-B$, an alternative characteristic length is the hydraulic diameter $d_{h}$. The values of $R e$ and $R o$ with $d_{h}$ as the characteristic length are shown in the parentheses.

\subsection{Dean and Coriolis Vortices}

An important feature relating to stabilization and destabilization is the appearance of the Dean and Coriolis vortices. It is of special interest because they may cause a significant change to flow structure and the wall heat transfer distribution. In addition, they have an important effect on transition from laminar to turbulent flow and relaminarization from turbulent to laminar flow.

The Dean and Coriolis vortices may be present as a result of centrifugal and Coriolis instabilities imposed on the flow by curvature and rotation. Experimentally, these vortices are identified by the mushroomshaped smoke patterns. Such patterns form because of spanwise variations of streamwise velocity due to the different inwash and outwash regions associated with pairs of counter-rotating vortices. The stems of individual mushroom shapes mark the region of flow away from the wall surface between the vortices which form one pair.
The possible various kinds of secondary instabilities of the Dean and Coriolis vortices may produce the Dean and Coriolis vortices with different features for different flow regions. The properties of disturbances triggering initial vortex formation (such as their steadiness, uniformness along the spanwise direction etc.) may also lead to various Dean and Coriolis vortices. Consequently, we may observe different mushroom-shaped smoke patterns due to single or combined effect of the two causes (secondary instabilities and initial disturbances). In a fully developed region which the present work is mainly concerned with, the Dean and Coriolis vortices become fully developed and should be less sensitive to the initial disturbances because once initiated, vortex behaviour and development are strongly controlled by the centrifugal and Coriolis instabilities from the curvature and rotation of the channel. The different Dean and Coriolis vortices observed in our experiments are, therefore, believed to be caused by their secondary instabilities.

The Dean and Coriolis vortices observed can be divided into two groups: symmetric and nonsymmetric vortices with respect to radial-streamwise plane. The symmetric Dean and Coriolis vortices appear usually at a dynamic parameter close to the critical value for their onset. Such vortices are identified by mushroom stems which form the radial symmetry lines. The mushroom-shaped smoke patterns are the mirror images on each side of the line. In other words, the two vortices in each pair are similar. The nonsymmetric Dean and Coriolis vortices are often observed at higher dynamic parameters. When viewed in cross (radial-spanwise) planes, such vortices are distorted generally by spanwise and/or radial unsteadiness in the flow such that no symmetric line is observed.

\subsubsection{Symmetric Coriolis Vortices}

Examples of smoke patterns from symmetric Coriolis vortex flow are shown in Fig. 4. They are obtained for $T s-B$ at $R e=193.6(352), R o=-1.171(-2.129)$ and $R e=452(822)$, $R o=-1.718(-3.124)$. The pat- 


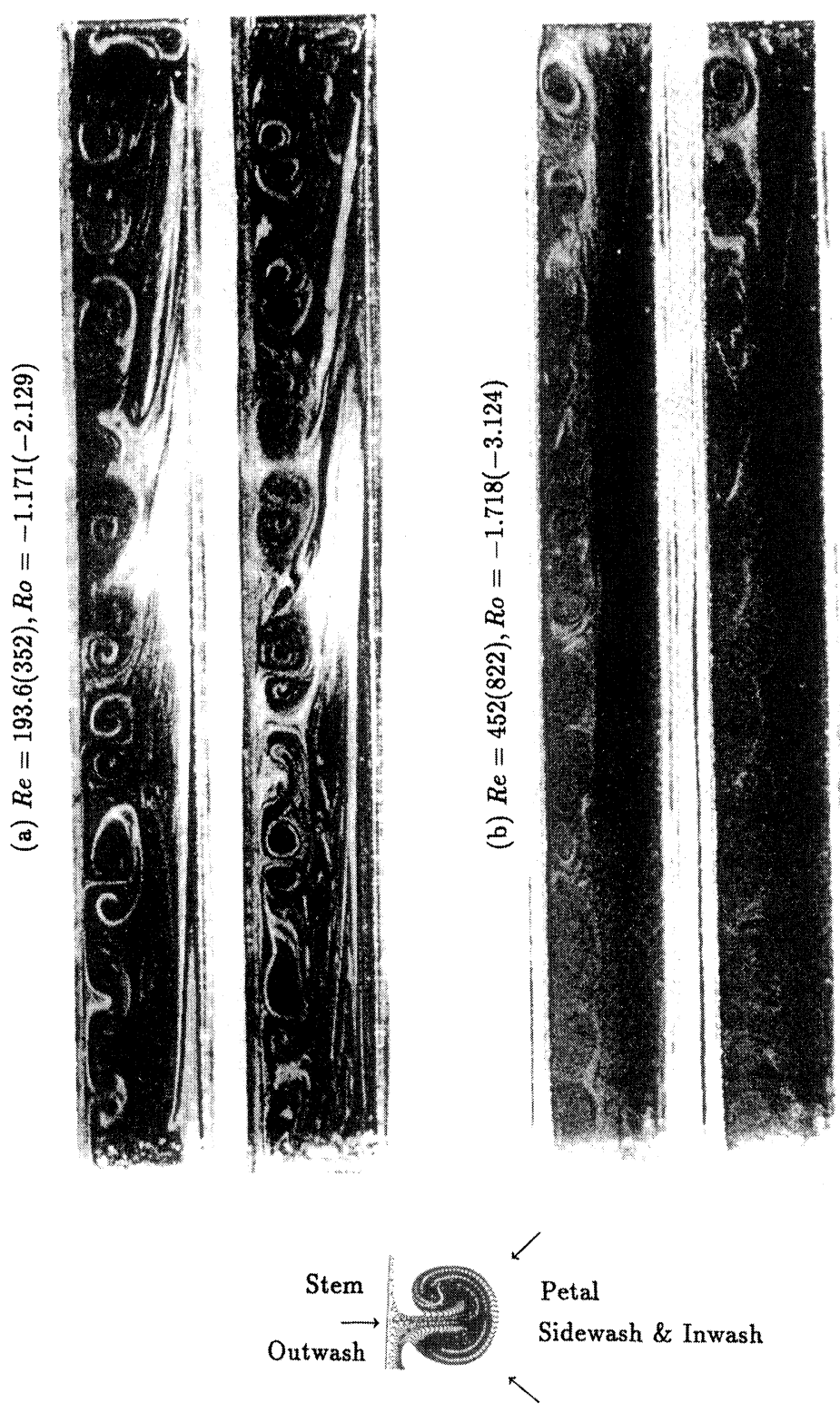

FIGURE 4 Symmetric Coriolis Vortices for $T s-B$.

terns are especially interesting because they show only small unsteadiness. No significant time variation on shape and size can be observed. The narrow mushroom stems indicate narrow outwash regions from the high pressure wall. Also the large mushroom petals imply large, spread-out sidewash and inwash regions. The fairly uniform size and shape at different span- wise positions indicate that the flow is fully developed since otherwise the difference will result from the sensitivity of developing vortices to initial disturbances.

A comparison of the smoke patterns in Fig. 4(a) with those in Fig. 4(b) shows that the shape of the symmetric Coriolis vortices changes with the operat- 
ing parameter. This is consistent with the numerical simulations (Finlay [1990]) which indicate that vortex shape changes with spanwise wavenumber, Reynolds number and rotation number.

\subsubsection{Radially Oscillating Coriolis Vortex Flow}

Two examples of radially oscillating Coriolis vortices are presented in Fig. 5. They occur at $R e=600, R o$ $=-1.37$ for $T s-A$ and at $R e=193.6(352), R o=$
$-0.663(-1.205)$ for $T s-B$ respectively. At this stage of flow, very little spanwise oscillation is present and mushroom patterns seem to move almost exclusively in the radial direction. When these oscillations occur, they are mostly symmetric Coriolis vortices, and the radial unsteadiness decreases in magnitude as the overall average vortex height increases.

The vortex heights at different spanwise locations in Fig. 5.(b) show relatively small variations in height and spacing. This indicates that the Coriolis vortices
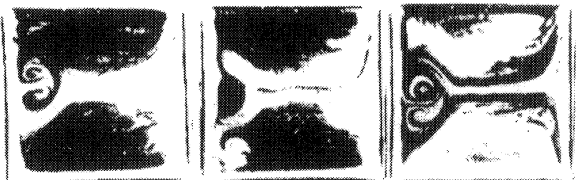

(a) $R e=600, R o=-1.37(T s-A)$
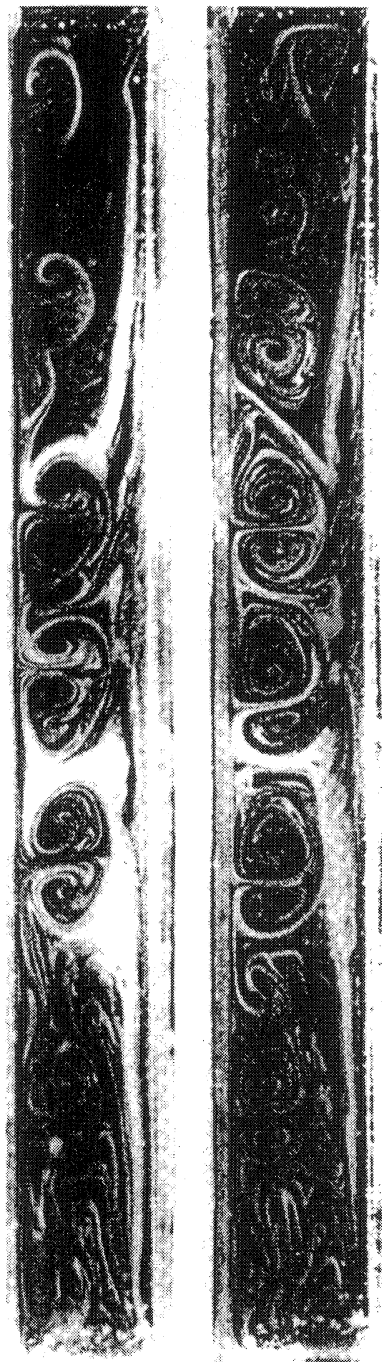

(b) $R e=193.6(352), R o=-0.663(-1.205)(T s-B)$ 
are fully developed along the streamwise direction and the oscillation is caused by the secondary instability of the Coriolis vortices.

\subsubsection{Spanwise Oscillating Dean and Coriolis Vortex Flow}

The mushroom-shaped smoke patterns at $R e=500$, $R o=0$ for $T s-A$ and at $R e=581(1056), R o=$ $-1.12(-2.036)$ for $T s-B$ are shown in Fig. 6. The time variations in shape show that very little radial oscillation is present and mushroom patterns seem to move almost exclusively in the spanwise direction. When these oscillations occur, they are mostly symmetric. The uniform height and spacing in spanwise direction provide evidence of fully developed vortices. As well, the oscillation is believed to be caused by the secondary instability.

\subsubsection{Simultaneous Spanwise and Radial Oscillating Dean Vortex Flow}

The Mushroom-shaped smoke patterns, oscillating simultaneously in the spanwise and radial directions may be observed without a twisting motion. One example is shown in Fig. 7 for $T s-A$ at $R e=550, R o=$ 0 . The vortices in this vortex flow are also nearly symmetric, and generally have larger-scale oscillation and greater unsteadiness than the smoke patterns identified with radial, span or twisting modes.

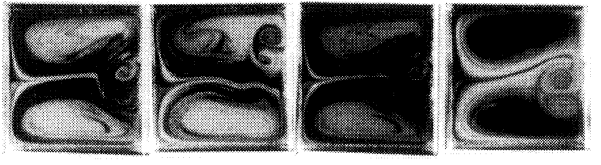

(a) Spanwise osclllating Dean vortices at $R e=500, R_{0}=0$ for $T s \sim A$

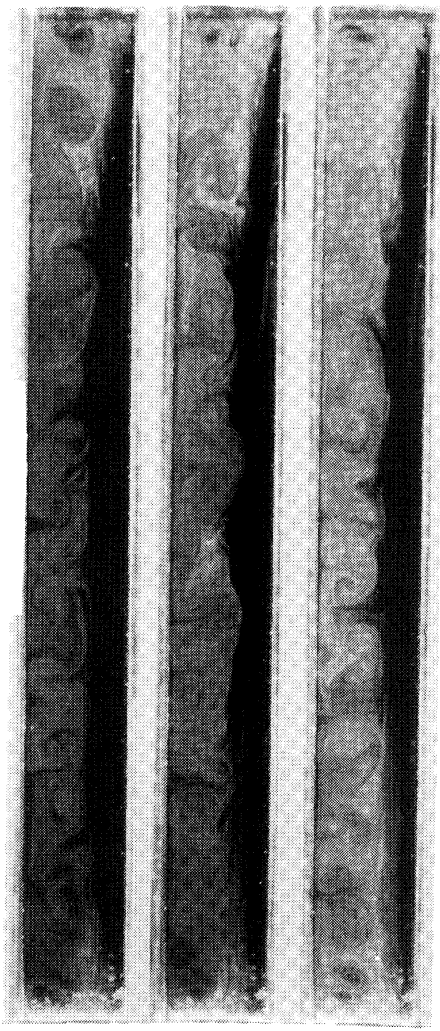

(b) Spanwise oscillating Coriolis vortices at $R e=581$ $(1056), R_{0}=-1.12(-2036)$ for $T_{s-B}$

FIGURE 6 Spanwise oscillating Dean and Coriolis vortices. 

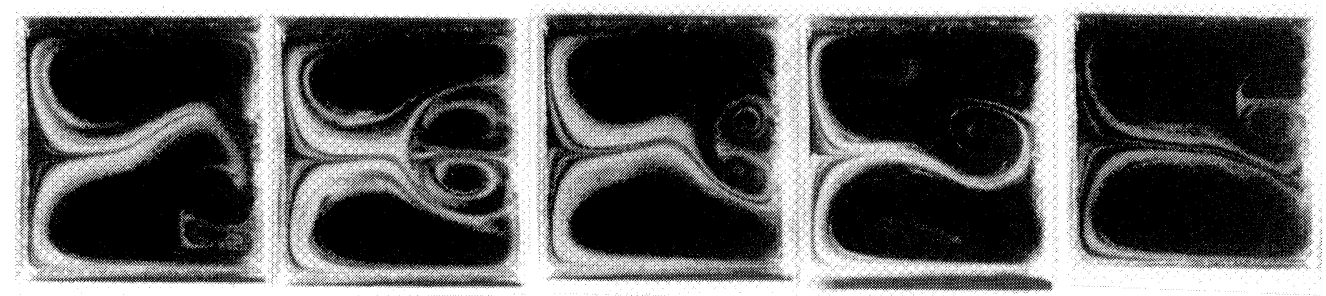

FIGURE 7 Simultaneous spanwise and radial oscillating Dean vortices at $R e=550$, Ro $=0$ for $T s-A$

\subsubsection{Twisting Coriolis Vortex Flow}

Finlay et al. [1988] and Finlay [1990] simulated curved and rotating channel flows using three-dimensional incompressible time-dependent Navier-Stokes equations. They found evidence that the Dean or Coriolis vortices develop two different types of travelling waves in the streamwise direction. The undulating Dean or Coriolis vortex flow contains long streamwise wavelengths and very small growth rates which make them difficult to observe experimentally. The twisting Dean or Coriolis vortex flow contains much shorter streamwise wavelength than the undulating Dean and Coriolis vortex flow. This causes them to be observed more readily.

Streamwise fully developed travelling waves generate same events at one time but different streamwise locations as at one streamwise location but different times. With twisting, the vortex centers oscillate only a little in the spanwise direction. The motion occurs mostly in the radial direction as the vortices in one pair oscillate in shape and strength.

The smoke patterns for twisting Dean and Coriolis vortices are mushroom-shaped with a rocking type of motion. Examples of smoke patterns from twisting Dean vortices are presented in Fig. 8. They were obtained at $R e=600, R o=0$ for $T s-A$ and at $R e=$ 581(1056), $R o=0(0)$ for $T s-B$. It is observed that the twisting Dean vortices are nonsymmetric. The Reynolds number for the two cases with twisting Dean vortices $(R o=0)$ are larger than $1.96 R e_{c}$ with $R e_{c}$ as the critical Reynolds number for the onset of the Dean vortices. These are consistent with the numerical simulations given by Finlay et al. [1988].

For $T s-B$, it is found that the rocking motion is rarely observed alone, rather it is usually observed in conjunction with at least one other mode of oscilla- tion. In many cases, the rocking vortex pairs are adjacent to pairs with a different type of motion.

\subsubsection{Small Vortex Pairs}

Using a linear stability theory and spectral method, Guo \& Finlay [1991] examined the Eckhaus stability of the Dean and Coriolis vortices. They found that the Eckhaus stability boundary is a small closed loop. Outside the boundary, the Eckhaus instability causes the vortex pair to split apart by the formation of a new vortex pair or merge together, and no vortex flow is stable to spanwise disturbances when $R e>$ $1.7 R e_{c}$. When $R e$ is not too high $\left(R e<4 R e_{c}\right)$, the wavenumber of vortices is selected by the Eckhaus instability. Similar phenomena have also been reported by Finlay et al. [1988], Finlay [1990] and Bland and Finlay [1991] in their numerical simulations of channel flows with curvature or rotation.

The vortex splitting/merging and readjustment of the spanwise wavenumber are clearly evidenced in the radial-span cross section by the presence of small vortex pairs at some instants. Examples are shown in Fig. 9 for $T s-B$ at $R e=452(822), R o=0(0)$; $R e=$ 452(822), $R o=-0.091(-0.165) ; R e=323.3(588)$, $R o=-0.819(-1.489)$ and $R e=581(1056), R o=$ $1.12(2.036)$ as pointed by an arrow.

\subsubsection{Complex Oscillating Models}

In some cases, usually with high dynamical parameters, the oscillation of the Dean and Coriolis vortices appear to be so chaotic such that the mushroomshaped patterns are barely recognizable or can not be identified clearly. Photographs of such patterns are 


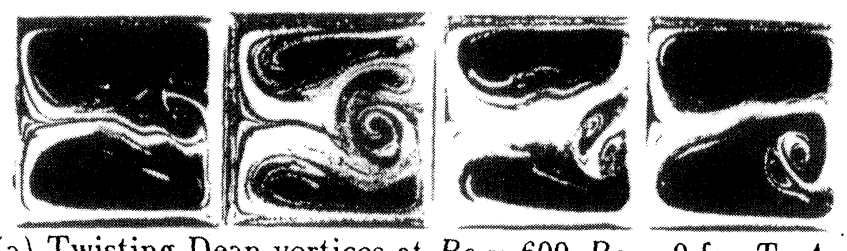

(a) Twisting Dean vortices at $R e=600, R_{o}=0$ for $T_{s-A}$
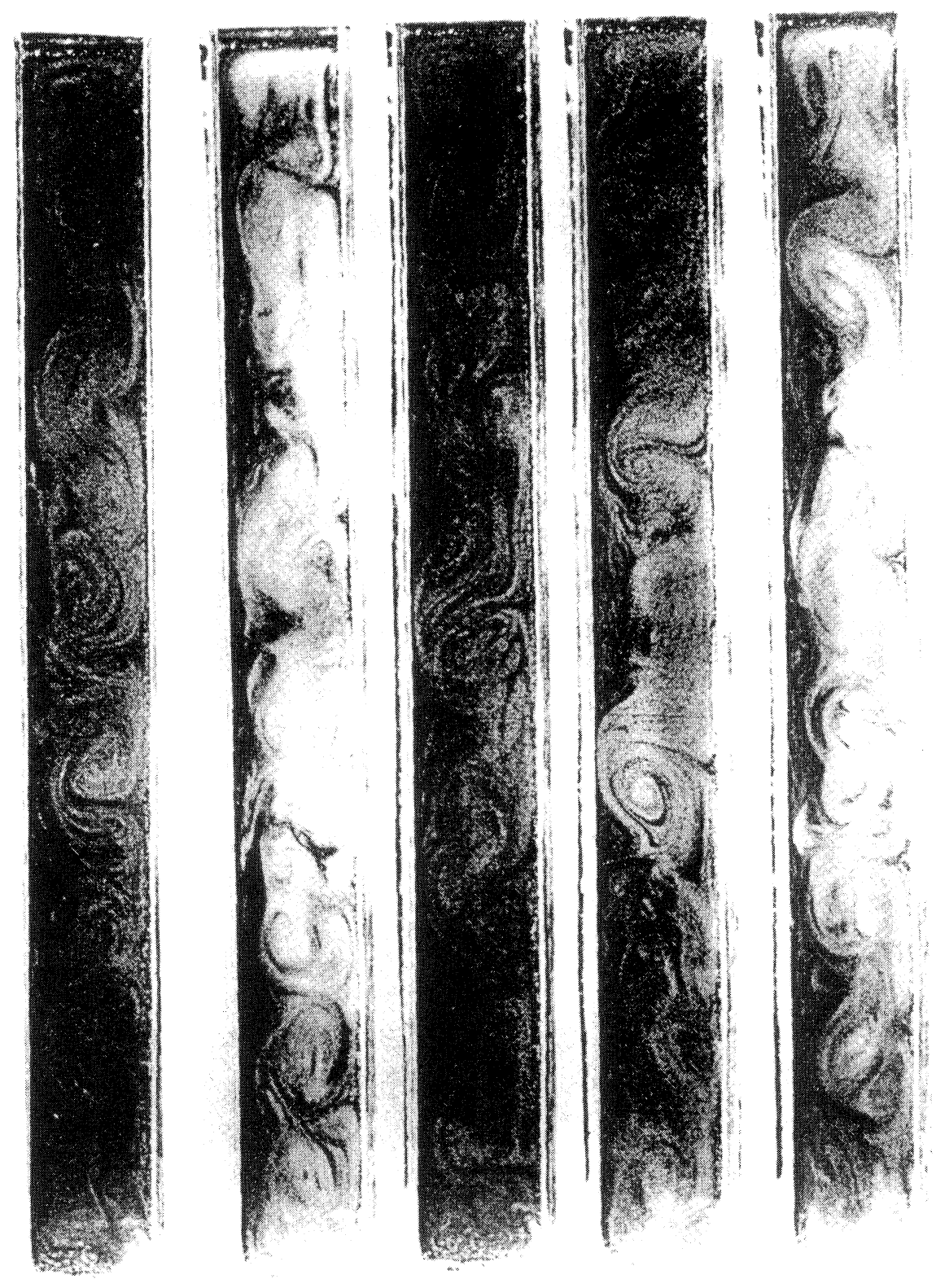

(b) Twisting Dean vortices at $R e=600(1056), R o=0(0)$ for $T s-B$

FIGURE 8 Twisting Dean vortices. 


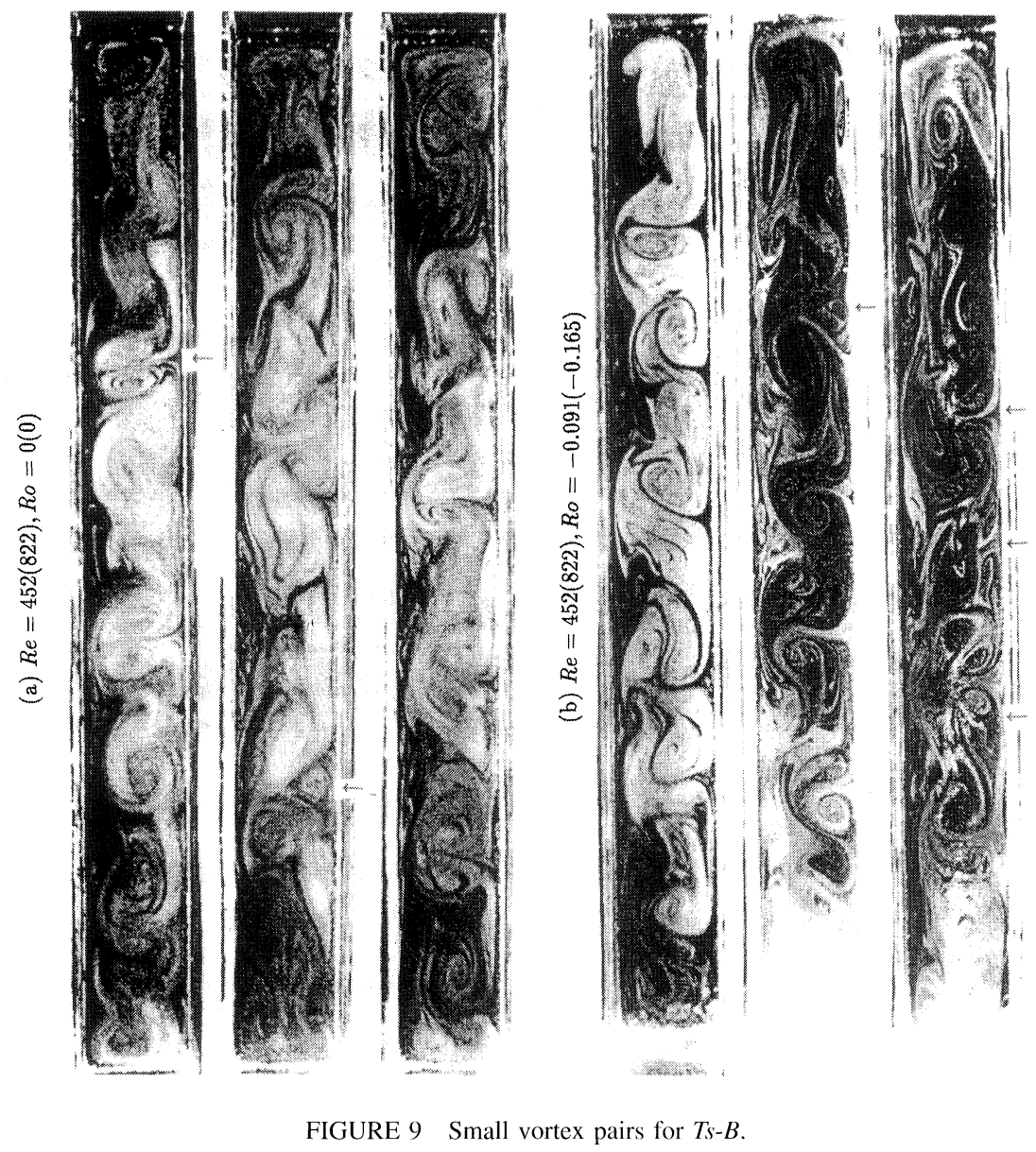

shown in Fig. 10 for $T s-B$ at $R e=193.6(352), R o=$ $0(0)$.

\subsection{Flows in Curved Channels with a High Rotation Rate}

A generalized Rayleigh stability criterion was derived by Wang and Cheng [1995] to analyze stable and unstable regions of flows with respect to the primary instability in a rotating curved channel of an infinite span. The result is

$$
\begin{aligned}
& \Pi=2\left[\frac{\sigma w}{1+\sigma(x-1 / 2)}+R o\right] \\
& {\left[\frac{d w}{d x}+\frac{\sigma W}{1+\sigma(x-1 / 2)}+2 R o\right]}
\end{aligned}
$$

where (see Fig. 1)

$$
x=\frac{X-R_{c}}{a} w=\frac{W}{W_{m}} R o=\frac{\Omega d}{W_{m}} \sigma=\frac{a}{R_{c}}
$$

The flow is stable in the flow domain with $\Pi>0$, and unstable in the domain with $\Pi<0$. This criterion was used to analyze the stable and unstable regions for the case with a small gap $(\sigma<<1)$ and a low rotation rate $(R o \sim o(\sigma))$. Here we extend the analysis to the case of a small gap with a high rotation rate.

For the small gap $(\sigma<<1)$ and high rotation rate $(R o \sim o(1))$, the generalized Rayleigh's criterion $\Pi$ can be approximated as

$$
\Pi(x)=2 R o\left(\frac{d w}{d x}+2 R o\right)
$$




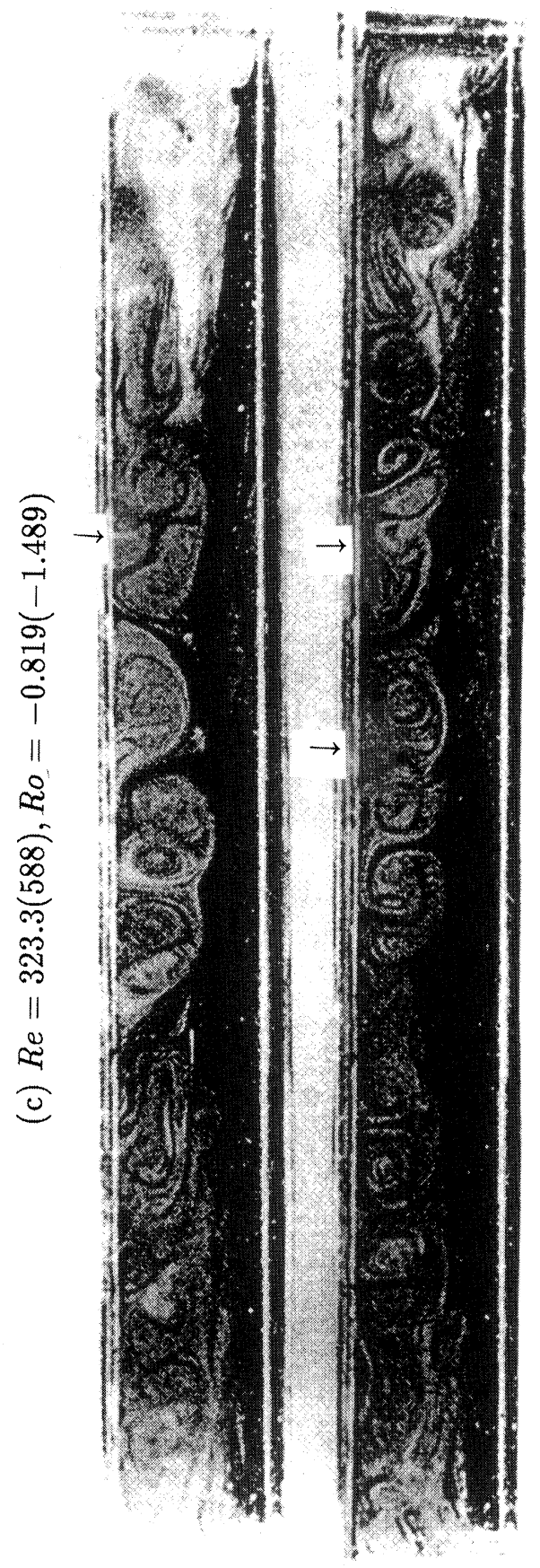

which vanishes at a radial position $x_{0}$ satisfying

$$
\frac{d w}{d x}+2 R o=0
$$

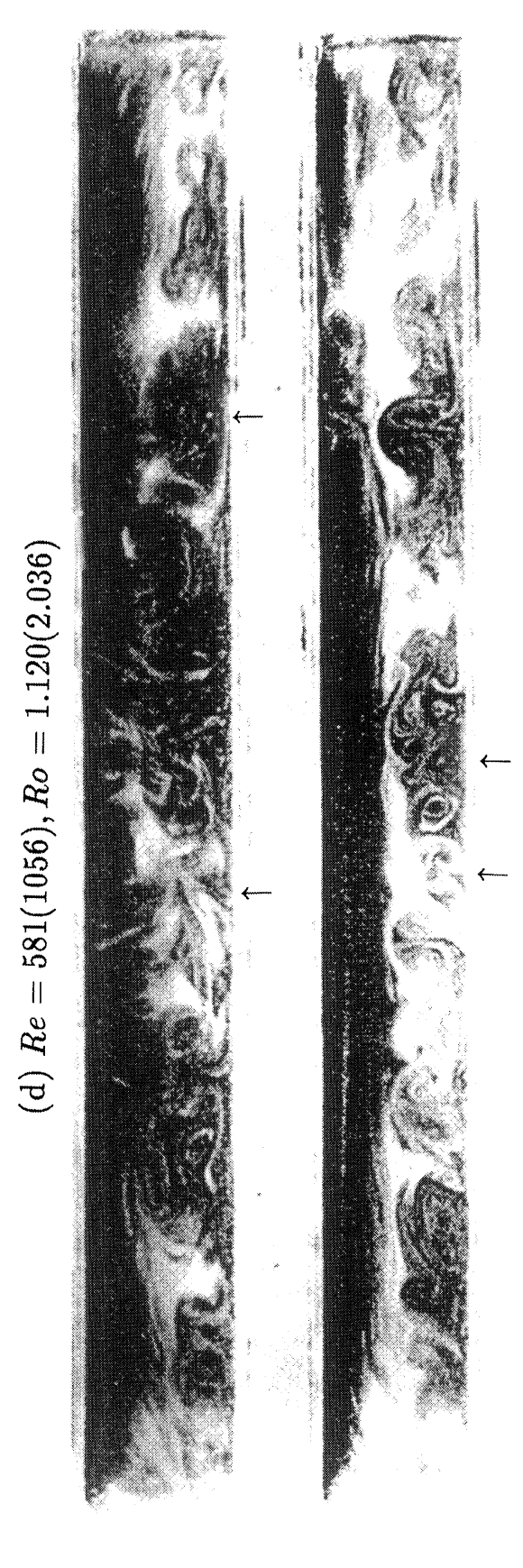

For the flow in a curved channel with rotation around the axis of curvature, the base flow can be written as (Chandrasekhar [1961]) 


$$
w(x)=6 x(1-x)
$$

This, with Eq.(6), yields

$$
x_{0}=\frac{1}{2}\left(1+\frac{R o}{3}\right) \quad \forall|R o| \leq 3
$$

The sign of $\Pi$ in different regions is summarized in Table I. The stable and unstable regions are shown in Fig. 11 (Although Table I and Fig. 11 show the results for the whole range of Ro, we should use the results in Wang and Cheng [1995] for the case with a low rotation rate). This shows that the unstable regions reduces as $|R o|$ increases, and the high rotation with $\left|R_{o}\right|>3$ always stabilizes the flow in the whole cross-section.

Examples of smoke patterns from flows in a region with $|R o|>3$ are illustrated in Fig. 12(a) for $T s-$ $A$ and in Fig. 12(b) for $T s-B$. They are obtained at $R e=200, R o=21.42 ; R e=200, R o=-10.05 ; R e$ $=600, R o=9.78$ and $R e=1000, R o=-3.43$ for $T s-A$ and at $R e=110(200), R o=-8.11(-14.75)$; $R e=110(200), R o=10.60(19.27) ; R e=452(822)$, $R o=-3.59(-6.53)$ and $R e=452(822)$, Ro := $3.45(6.27)$ for $T s-B$. The patterns are seen to be somewhat similar to the bursting flow in the turbulent boundary layer (Kline et al. [1967]), and are far from the one pair of counterrotating vortices predicted by Ludwieg [1951] and Hocking [1967]. Therefore, the boundary layer approximation may not be valid for flow in the curved channels with a high rotation rate. Also the high unsteadiness of flow implies that the flow is controlled by the secondary instability rather than the primary instability. In particular, the secondary instability can produce the low Reynolds number turbulent flows.

\section{CONCLUDING REMARKS}

An experimental apparatus was designed and constructed especially to visualize the fully developed flows in two kinds of channels with streamwise curvature and spanwise rotation in terms of the end-view secondary flow near the exit of the test sections. The
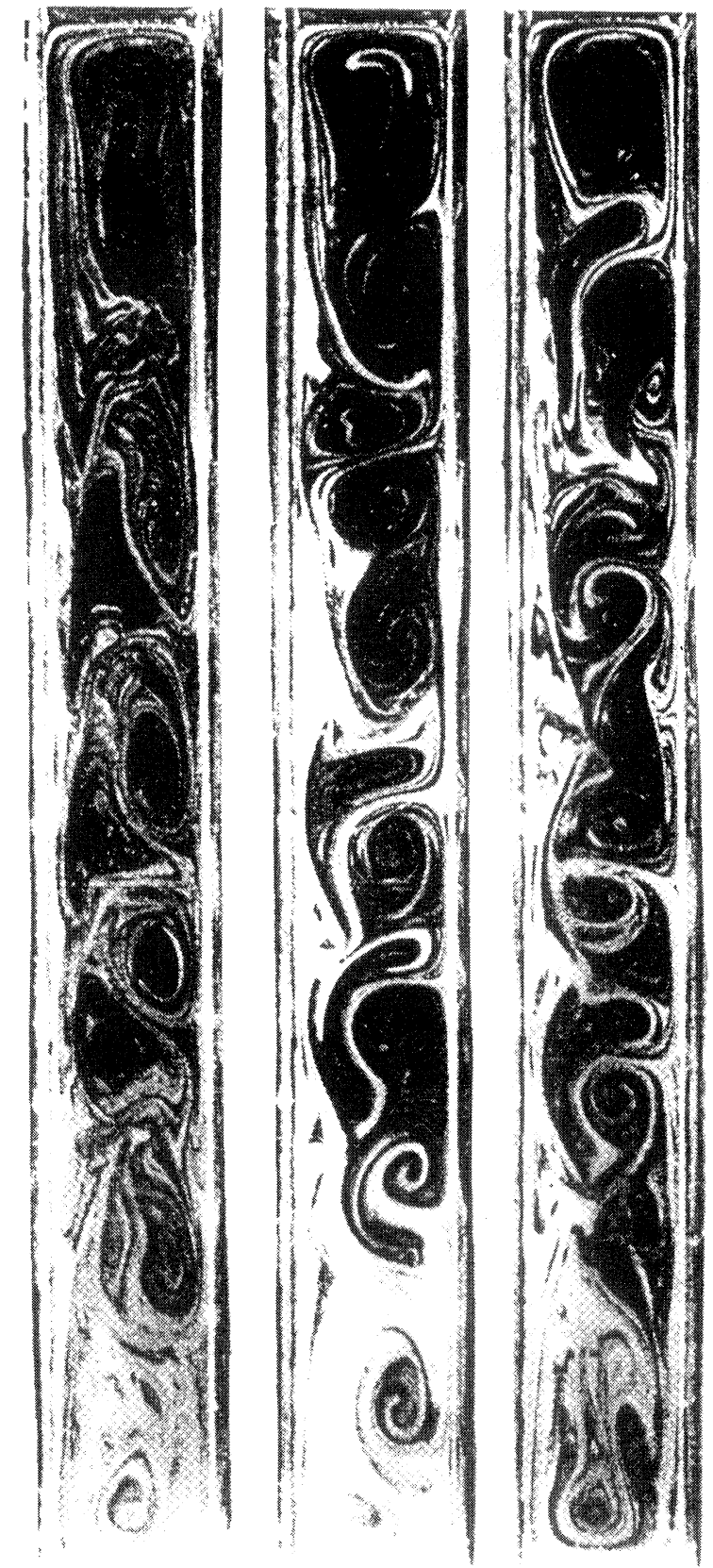

FIGURE 10 Complex oscillating Dean vortices at $R e=$ 193.6(352), $R o=0(0)$ for $T s-B$.

work focuses on the Dean and Coriolis vortices under the effects of the secondary instability, and the flows in the region with $\left|R_{O}\right|>3$. 
TABLE I Sign of II for the case of small gap and high rotation rate

\begin{tabular}{lcc}
\hline Ro & Region with Positive II & Region with Negative II \\
\hline Ro $\leq-3$ & $0<x<1$ & $0<x<x_{0}$ \\
$-3<R o \leq 0$ & $x_{0}<x<1$ & $x_{0}<x<1$ \\
$0 \leq R o<3$ & $0<x<x_{0}$ & \\
$R o \geq 3$ & $0 \leq x \leq 1$ & \\
\hline
\end{tabular}

Pairs of counter-rotating Dean and Coriolis vortices are observed as the mushroom-shaped smoke patterns. The narrow mushroom stems indicate the narrow outwash flow regions while large mushroom petals imply the large, spread-out sidewash and inwash regions. The fairly steady, symmetric Dean and Coriolis vortices are observed in some parameter regions. Their size and shape change with the operating parameters.

Secondary instabilities of the Dean and Coriolis vortices lead to oscillation in various forms even in the streamwise fully developed flow regions. The observed oscillating modes include: (1) one with mostly radial motion, (2) one with mostly spanwise motion, (3) one with significant simultaneous radial and spanwise motion, and (4) one with rocking motion. In addition, the oscillating modes are also present with the motion so complex that it is rather difficult to describe the flow visualization result. The experimental results are to be confirmed both theoretically and numerically in the future.

In the cross planes, the twisting Dean and Coriolis vortices are evidenced by nonsymmetric mushroomshaped smoke patterns with rocking motion. The region where the twisting Dean and Coriolis vortices are observed is in agreement with that predicted by Finlay et al. [1988] and Finlay [1990]. This kind of vortex flow differs from that with simultaneous radial and spanwise motion mainly in three aspects: (1) the twisting vortices are nonsymmetric but the simultaneous radial and spanwise oscillating vortices are symmetric, (2) with twisting, the spanwise locations of vortex centers oscillate only a little. With simultaneous radial and spanwise oscillating, the spanwise

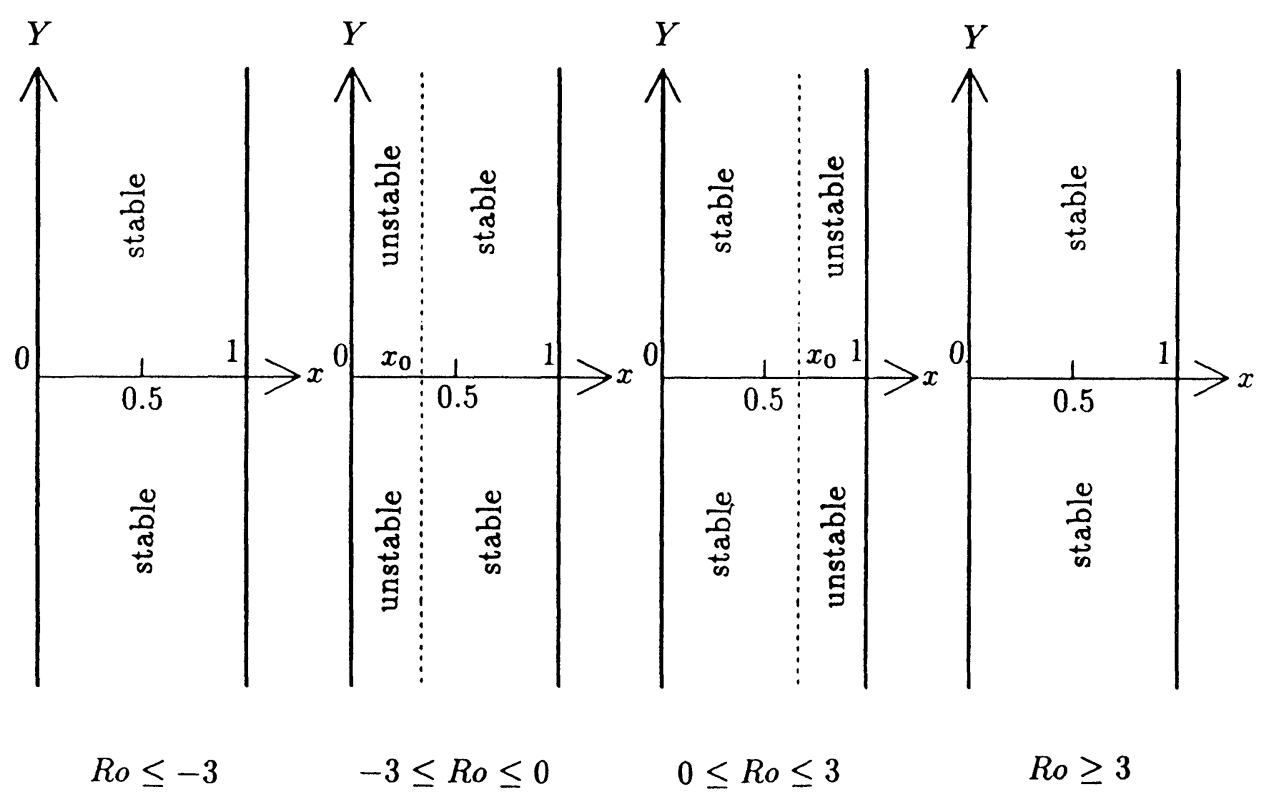

FIGURE 11 Stable and unstable regions for the case of a small gap with a high rotation rate. 


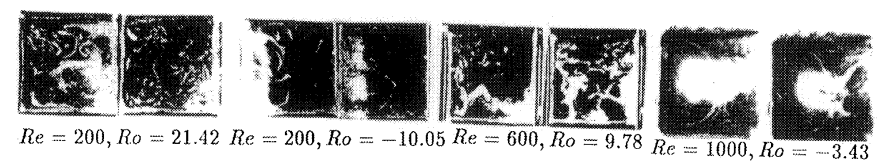

(a) Flows in a region with $|R o|>3 \mid$ for $T S$ s $A$
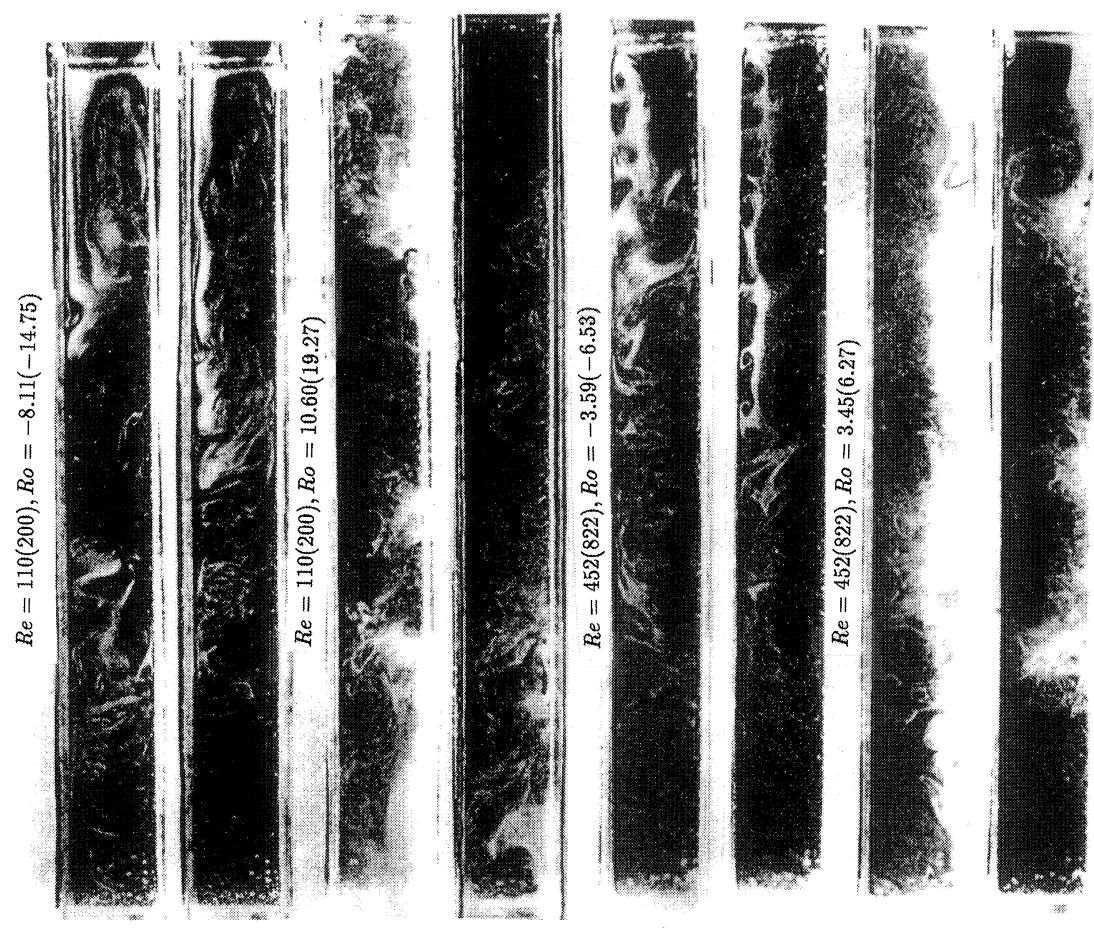

(b) Flows in a region with $\left|R_{0}\right|>3 \mid$ for $T s-B$

FIGURE 12 Flows in a region with $|R o|>3 \mid$.

locations of vortex centers can oscillate significantly, and (3) the simultaneous radial and spanwise oscillating Dean and Coriolis vortices usually have largerscale oscillation and greater unsteadiness than the twisting Dean and Coriolis vortices.

The vortex pairs sometime seem to appear and disappear as indicated by small vortex pairs observed temporarily in the cross planes for some experimental conditions. This is believed to be caused by the Eckhaus instability discussed by Guo and Finlay [1991].

The flow at large $|R o|$ is found to be controlled by the secondary instability rather than the primary instability. In particular, the secondary instability leads the flow with large $|R o|$ to be unsteady and turbulent somewhat like the bursting flow in the turbulent boundary layer. This appears to produce the low Reynolds number turbulent flow and one may conclude that the boundary layer approximation is not valid for this type of flow.

\section{Acknowledgement}

This work was supported by the Natural Sciences and Engineering Research Council of Canada through an operating grant. 


\section{References}

Bland, S. B. and Finlay, W. H. (1991) Transitions towards Turbulence in a Curved Channel, Phys. Fluids, Vol. A3, pp. 106-114

Chandrasekhar, S. (1961) Hydrodynamic and Hydromagnetic Stability, Oxford University Press, London

Daskopoulos, P. and Lenhoff, A. M. (1990) Flow in Curved Ducts: Part 2, Rotating Ducts, J. Fluid Mech., Vol. 217, pp. 575-593

Finlay, W. H. (1990) Transition to Oscillatory Motion in Rotating Channel Flow, J. Fluid Mech., Vol. 215, pp. 209-227

Finlay, W. H., Keller, J. B. and Ferziger, J. H. (1988) Instabilities and Transition in Curved Channel Flow, J. Fluid Mech., Vol. 194, pp. 417-456

Greenspan, H. P. (1968) The Theory of Rotating Fluids, Cambridge University Press, London

Guo, Y. and Finlay, W. H. (1991) Splitting, Merging and Wavelength Selection of Vortices in Curved and/or Rotating Channel Flow due to Eckhaus Instability, J. Fluid Mech., Vol. 228, pp. 661-691.

Hochrainer, D. (1971) A New Centrifuge to Measure the Aerodynamic Diameter of Aerosol Particles in the Submicron Range, $J$. Colloid Interface Sci., Vol. 36, pp. 191-194.

Hocking, L. M. (1967) Boundary and Shear Layers in a Curved Rotating Pipe, J. Math. Phys. Sci., Vol. 1, pp. 123-136.

Hoover, M. D., Stöber, W. and Morawietz, G. (1984) Experiment on Laminar Flow in a Rotating, Curved Duct of Rectangular Cross Section, J. Fluids Engi., Vol. 106, pp. 38-44.

Ito, H. and Motai, T. (1974) Secondary Flow in a Rotating Curved Pipe, Rep. Inst. High Speed Mech., Vol. 29, 33-57.

Kline, S. J., Reynolds, W. C., Schraub, F. A. and Runstadler, P. W. (1967) The Structure of Turbulent Boundary Layers, J. Fluid Mech., Vol. 30, pp. 741-773.

Kotrappa, P. and Light, M. E. (1972) Design and Performance of the Lovelace Aerosol Particle Separator, Review of Scientific Instruments, Vol. 43, pp. 1106-1112.

Lennartz, J. W., Gorensek, M. B. and Adler, R. J. (1987) Separation of Fine-Particle Dispersions Using Periodic Flows in a Spinning Coiled Tube. Part III: Batch Fractionation Experiments, AICH E J., Vol. 33, pp. 506-509.

Ludwieg, H. (1951) Die Ausgebildete Kanalströmung in Einem Rotierenden System, Ing.-Arch. Bd., Vol. 19, pp. 296-308.
Miyazaki, H. (1971) Combined Free and Forced Convective Heat Transfer and Fluid Flow in a Rotating Curved Circular Tube. Int. J. Heat Mass Transfer, Vol. 14, pp. 1295-1309.

Miyazaki, H. (1973) Combined Free and Forced Convective Heat Transfer and Fluid Flow in a Rotating Curved Rectangular Tube, J. Heat Transfer, Vol. 95, pp. 64-71.

Morris, W. D. (1981) Heat Transfer and Fluid Flow in Rotating Coolant Channels, Research Studies Press, Wiley, New York.

Nandakumar, K., Raszillier, H. and Durst, F. (1991) Flow through Rotating Rectangular Ducts, Phys. Fluids, Vol. A3, pp. 770-781. Papanu, J. S., Adler, R. J., Gorensek, M. B. and Menon, M. M. (1986) Separation of Fine Particle Dispersions Using Periodic Flows in a Spinning Coiled Tube, AICH E J., Vol. 32, pp. 798808.

Qiu, L., Wang, L. and Sun, Y. (1990) Heat Transfer from Tubes which Extend Radially Outward from a Rotating Shaft, in Heat Transfer Enhancement and Energy Conservation, Eds. S. J. Deng, T. N. Veziroğlu, Y. K. Tan and L. Q. Chen, pp. 231-236, Hemisphere, Washington, DC.

Selmi, M., Nandakumar, K. and Finlay, W. H. (1994) A Bifurcation Study of Viscous Flow through a Rotating Curved Duct, J. Fluid Mech., Vol. 262, pp. 353-375.

Stöber, W. and Flachsbart, H. (1969) Size-Separating Precipitation of Aerosols in a Spinning Spiral Duct, Environmental science and technology, Vol. 3, pp. 1280-1296.

Wang, L. (1995) Flow and Heat Transfer in Rotating Curved Channels, Ph.D. Dissertation, Dept. of Mech. Eng., University of Alberta.

Wang, L. and Cheng, K. C. (1995) Flow in Curved Channels with a Low Negative Rotation Speed, Physical Review E, Vol. 51, pp. 1155-1161.

Wang, L. and Cheng, K. C. (1996a) Flow Transitions and Combined Free and Forced Convective Heat Transfer in a Rotating Curved Circular Tube, Int. J. Heat Mass Transfer, Vol. 39, pp. 3381-3400.

Wang, L. and Cheng, K. C. (1996b) Flow Transitions and Combined Free and Forced Convective Heat Transfer in Rotating Curved Channels: The Case of Positive Rotation, Phys. Fluids, Vol. 8, pp. 1553-1573. 


\section{ait \\ ENERGY MATERIALS}

M A N E Y publishing

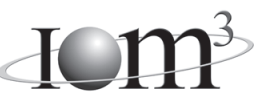

\section{Materials Science \& Engineering for Energy Systems}

Maney Publishing on behalf of the Institute of Materials, Minerals and Mining

The Institute of Materials, Minerals \& Mining

Economic and environmental factors are creating ever greater pressures for the efficient generation, transmission and use of energy. Materials developments are crucial to progress in all these areas: to innovation in design; to extending lifetime and maintenance intervals; and to successful operation in more demanding environments. Drawing together the broad community with interests in these areas, Energy Materials addresses materials needs in future energy generation, transmission, utilisation, conservation and storage. The journal covers thermal generation and gas turbines; renewable power (wind, wave, tidal, hydro, solar and geothermal); fuel cells (low and high temperature); materials issues relevant to biomass and biotechnology; nuclear power generation (fission and fusion); hydrogen generation and storage in the context of the 'hydrogen economy'; and the transmission and storage of the energy produced.

As well as publishing high-quality peer-reviewed research, Energy Materials promotes discussion of issues common to all sectors, through commissioned reviews and commentaries. The journal includes coverage of energy economics and policy, and broader social issues, since the political and legislative context influence research and investment decisions.

\section{CALL FOR PAPERS}

Contributions to the journal should be submitted online at http://ema.edmgr.com

To view the Notes for Contributors please visit: www.maney.co.uk/journals/notes/ema

Upon publication in 2006, this journal will be available via the Ingenta Connect journals service. To view free sample content online visit: www.ingentaconnect.com/content/maney

For further information please contact:

Maney Publishing UK

Tel: +44 (0)113 2497481 Fax: +44 (0)1132486983 Email: subscriptions@maney.co.uk

or

Maney Publishing North America

Tel (toll free): 8662975154 Fax: 6173546875 Email: maney@maneyusa.com

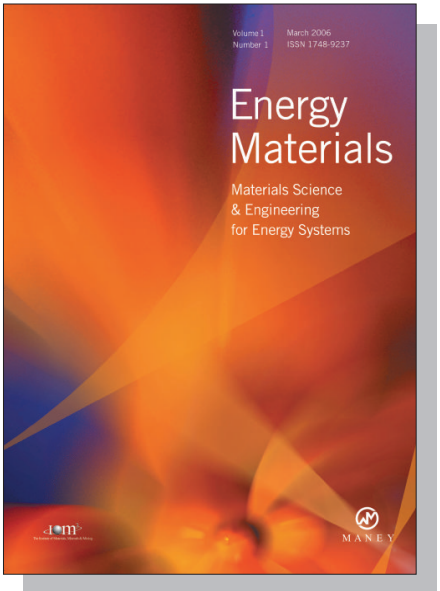

EDITORS

Dr Fujio Abe

NIMS, Japan

Dr John Hald, IPL-MPT, Technical University of Denmark, Denmark

Dr R Viswanathan, EPRI, USA

\section{SUBSCRIPTION INFORMATION}

Volume 1 (2006), 4 issues per year

Print ISSN: 1748-9237 Online ISSN: 1748-9245

Individual rate: $£ 76.00 / U S \$ 141.00$

Institutional rate: $£ 235.00 /$ US $\$ 435.00$

Online-only institutional rate: $£ 199.00 / U S \$ 367.00$

For special $\mathrm{IOM}^{3}$ member rates please email

subscriptions@maney.co.uk

\section{For further information or to subscribe online please visit www.maney.co.uk}



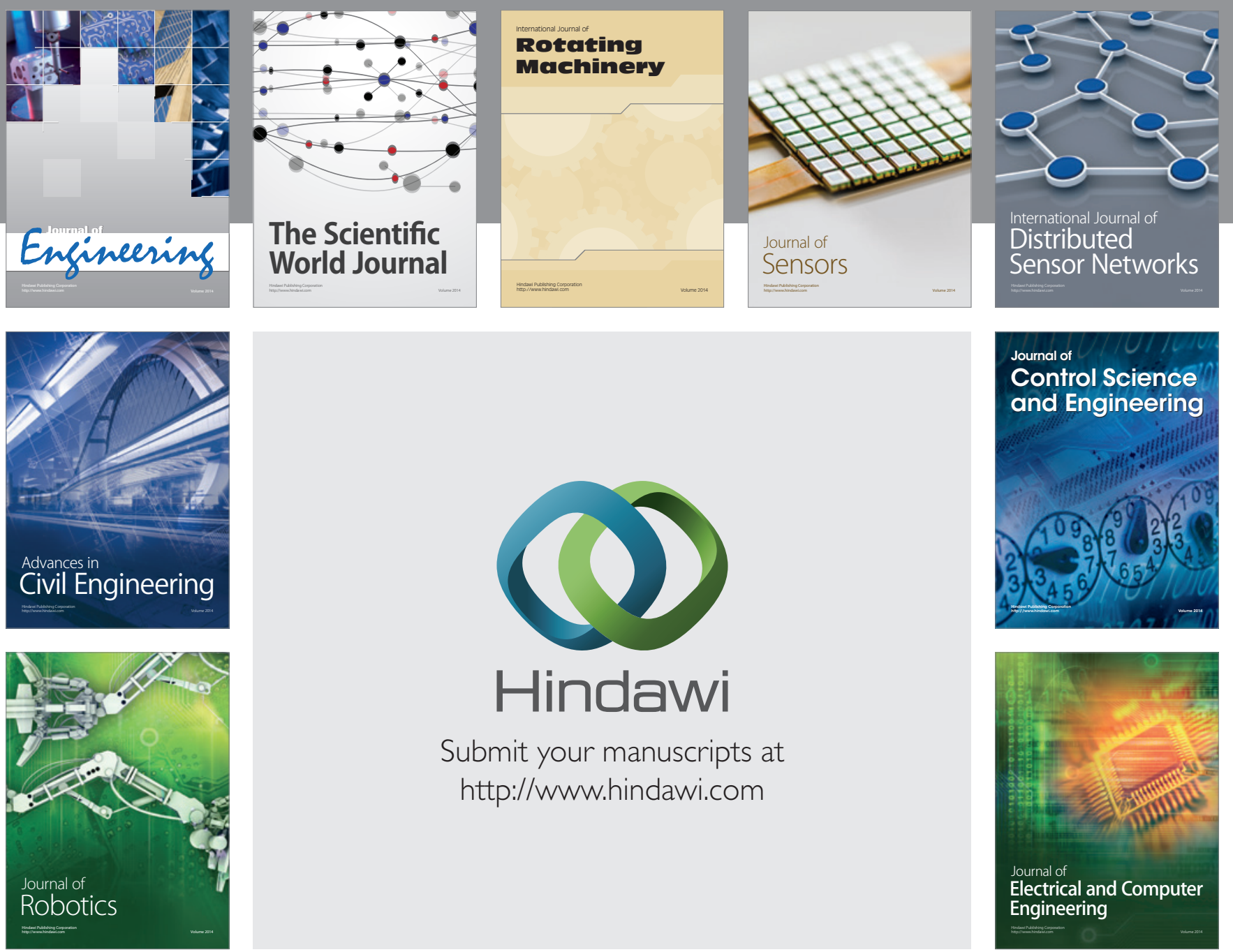

Submit your manuscripts at

http://www.hindawi.com
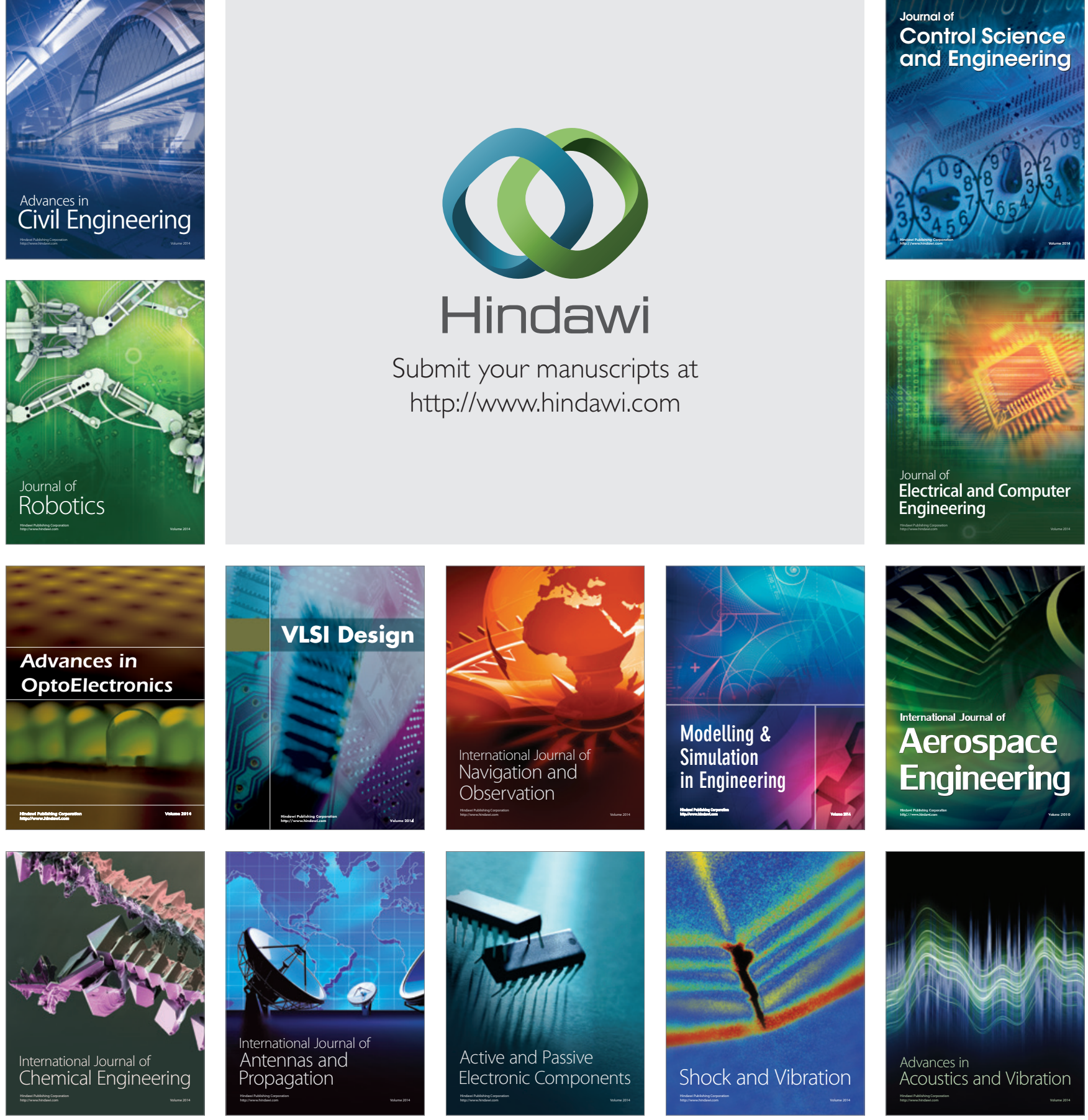